\title{
Análise Setorial do Impacto da Política dos APLs no Rio Grande do Sul
}

\author{
Suamy Cristina Savaris Linhares \\ Universidade Federal de Pelotas \\ André Carraro \\ Universidade Federal de Pelotas
}

\begin{abstract}
Resumo
Este trabalho avaliou a política de Arranjos Produtivos Locais (APL) no Rio Grande do Sul. Foi analisado se os setores dos municípios que possuem APL tiveram algum impacto sobre o nível de emprego e renda em relação àqueles que não possuem. Os dados foram retirados do site da RAIS utilizando-se 672 classes CNAE 2.0 para todos os municípios do estado no período de 2006 a 2014. O método utilizado foi o de diferenças-em-diferenças aplicando-se matching e efeito-fixo para os setores e o tempo. O arranjo Moveleiro da Serra Gaúcha e o APL de Pedras, Gemas e Joias foram os únicos que obtiveram resultados positivos e significativos para o nível de emprego. Na análise da remuneração média, nove APLs apresentaram efeitos positivos e com significância.
\end{abstract}

Palavras Chaves: APL. Economia regional. Rio Grande do Sul.

\section{Local Productive Arrangements: A Strategy for Better Performance}

\begin{abstract}
This study evaluates the Local Productive Arrangements (LPAs) policy in Rio Grande do Sul. It will be examined was analyzed whether the sectors of the municipalities that have APL had some impact on the level of employment and income than those who don't. The data are taken from the RAIS using 672 CNAE 2.0's classes site for all municipalities in the state from 2006 to 2014. The method used was the difference-in-differences applying matching and fixed-effect for the sectors and time. The arrangement named Moveleiro da Serra Gaúcha and the arrangement named Pedras, Gemas e Joias were the ones who had positive and significant results for the level of employment. On average remuneration analysis, nine APLs had positive and significant effects.
\end{abstract}

Keywords: LPA. Regional economy. Rio Grande do Sul.

\section{Arreglos Producivos Locales: Estrategia para um Mejor Desempeño}

\section{Resumen}

Este trabajo evaluó la política de Arreglos Productivos locales (APL) para los municipios de Rio Grande do Sul. Se analizó el impacto en los sectores de los municipios que poseen APL en 
relación a aquellos que no poseen en el período de 2006 a 2014. El arreglo Mueble de la Sierra Gaucha y el APL de Piedras, Gemas y Joias fueron los únicos que obtuvieron resultados positivos y significativos para el nivel de empleo. En el análisis de la remuneración media, nueve APL presentaron efectos positivos.

Palabras clave: APL. Economía Regional. Rio Grande do Sul.

\section{INTRODUÇÃO}

As duas últimas décadas apresentaram notório crescimento no interesse pelo papel dos Arranjos Produtivos Locais (APLs) na promoção do desenvolvimento regional. O tema passou a frequentar a agenda acadêmica (BOURLEGAT, 2006; COSTA, ANDRADE e SILVA, 2006; KIRSCHBAUM, CARVALHO e BRITO, 2007; LIGO et. al., 2007; entre outros) e ganhou espaço também na agenda política (MDIC, 2013; SPDI, 2013) por estar associado a uma solução factível para o desenvolvimento econômico, principalmente de regióes relativamente estagnadas economicamente.

O objetivo deste artigo é realizar uma avaliação empírica inicial do incentivo público ao desenvolvimento de APLs no estado do Rio Grande do Sul. Especificamente, pretende-se avaliar o impacto sobre as variáveis de emprego e remuneração média dos setores onde há arranjos produtivos locais identificados. De uma forma geral, os estudos sobre APLs no Brasil possuem um viés mais qualitativo. Nessa linha destacam-se Bourlegat (2006); Costa, Andrade e Silva (2004); Ligo et al. (2007) e Tatsch e Passos (2008). Embora a abordagem qualitativa seja relevante, é também necessário e importante, tanto em termos acadêmicos como em termos pragmáticos, subsidiar o formulador de políticas públicas com avaliações do impacto econômico que lhe permitam identificar casos de sucesso ou necessidades de mudanças, de tal forma a obter o resultado esperado quando do desenho do incentivo político. O formulador de políticas públicas precisa ter em mãos uma análise aprofundada da situação dos arranjos em todas as suas esferas, sejam elas econômicas, governamentais ou sociais.

Esta é a lacuna na literatura nacional que este trabalho pretende suprir. Contudo, ele não tem a pretensão de dar uma resposta definitiva sobre qual é o papel do Arranjo produtivo Local no crescimento econômico, ou se ele passou a ser um indutor do crescimento econômico, mas de apresentar evidências empíricas que possam estimular novos trabalhos de avaliação de impacto econômico em políticas públicas de impacto regional. Especificamente este trabalho avalia o impacto dos APLs apoiados pela Agência Gaúcha de Desenvolvimento e Promoção do Investimento (AGDI) sobre o nível de renda do trabalhador e sobre o nível de emprego. A hipótese central deste trabalho é a de que os APLs impactaram positivamente sobre o emprego e a renda dos setores dos munícipios gaúchos.

O trabalho está dividido em cinco seções. A seção dois apresenta o modelo teórico de Estrutura-Conduta Desempenho os principais conceitos que envolvem o tema e algumas evidências empíricas. A seção três expõe a origem da base de dados e a metodologia utilizada para a análise do impacto. Os resultados estimados estão na seção quatro. Por fim, a seção cinco traz as considerações finais do trabalho. 


\section{A ESTRUTURA DE MERCADO E OS ARRANJOS PRODUTIVOS LOCAIS}

Uma estratégia competitiva que as empresas de um mesmo setor se utilizam é a aglomeração como forma de obter maior competitividade no mercado. Ao atentar para a estrutura, elas buscam um desempenho econômico melhor via ajuste na conduta de suas ações: o modelo estrutura-conduta-desempenho (ECD) que ajuda a entender o comportamento estratégico que está presente na decisão de aglomeração das empresas de um mesmo setor em um arranjo produtivo. A seguir tal modelo é descrito para, posteriormente, subsidiar uma discussão sobre arranjos produtivos locais a ser apresentada.

\subsection{O Modelo Estrutura-Conduta-Desempenho}

O modelo estrutura-conduta-desempenho (ECD) é um paradigma de análise para utilizar o conceito de competitividade empresarial. Para que o conceito possa ser corretamente operacionalizado é necessário que os principais elementos-chave do ambiente interno que irão determinar a estrutura do mercado, a conduta dos empresários e o desempenho estejam incorporados no modelo de análise (SCHERER e ROSS, 1990).

O paradigma ECD estabelece que o desempenho competitivo (rentabilidade) está relacionado com a estrutura de mercado (nível de concentração industrial). A conduta empresarial é o canal que determinará seu melhor ou pior desempenho. Ou seja, por meio do seu comportamento em relação à política de vendas e fixação de preços, bem como a de decisão de aliar-se ou não com outros empresários do setor irá interferir na dinâmica de seu crescimento e de sua lucratividade (TIROLE, 1988).

Dentro desse paradigma aborda-se o conceito de competitividade sistêmica que é mais consistente que o conceito de competitividade convencional por ser mais amplo ao não se limitar aos fatores internos da empresa como as economias de escala e escopo. O conceito vai além ao adicionar variáveis externas que influenciam a empresa como a infraestrutura econômica, a infraestrutura social, a política macroeconômica bem como as políticas setoriais e regionais, entre outras como o desenvolvimento de capital humano e as políticas de fomento, geração e difusão de inovações tecnológicas (POSSAS, 1999).

Ao estar atento a toda a cadeia de negócios, à modernização das instituições, o empresário busca a melhor estratégia, a melhor conduta, para preservar a competitividade de sua empresa ao longo do tempo. O objetivo de considerar a abordagem sistêmica é compreender a dinâmica das variáveis macroeconômicas, microeconômicas, institucionais, sociais e políticas, cujo resultado pode ser alcançar vantagens competitivas necessárias para criar, manter e/ou ampliar a participação da empresa no seu mercado (POSSAS,1999; POSSAS, 2002).

Os fatores sistêmicos que determinam a competitividade em uma economia moderna têm na sua origem, segundo Carvalho et al. (2006), três níveis: fatores sistêmicos regulatórios que estimulam o ambiente competitivo; os fatores infra estruturais que provêm externalidades positivas capazes de aperfeiçoar a competitividade empresarial; e os fatores sistêmicos político-institucionais, que são as instituições sociais e governamentais que afetarão os planos dos empresários. 
O que se observa a partir do paradigma ECD é que os Arranjos Produtivos Locais podem ser representados como uma estratégia empresarial, em a aglomeração e cooperação entre os empresários são a conduta que busca, junto aos fatores sistêmicos, um melhor desempenho do setor. A seção seguinte define melhor os APLs e apresenta alguns casos que parecem confirmar essa estratégia.

\subsection{Arranjos Produtivos Locais: estratégia para um melhor desempenho}

A literatura internacional aborda o assunto de aglomerações de empresas através de diferentes tipologias. O termo usualmente utilizado é o "cluster", porém, foram encontrados alguns trabalhos com políticas governamentais muito semelhantes à política do APL. Assim, faz-se necessário conceituar os termos que são comumente encontrados na literatura como os clusters, sistemas produtivos/inovativos locais, distritos industriais e arranjos produtivos locais.

O conceito de cluster passou ter maior utilização a partir dos trabalhos feitos por Porter (1993). Segundo este autor:

cluster é um grupo geograficamente próximo de companhias
interconectadas e instituições associadas num ramo particular,
interligados por pontos em comum e complementares. O escopo
geográfico de um cluster pode ser uma única cidade ou estado ou um país
ou até mesmo um vínculo de países vizinhos. (PORTER, 1998, p.199).

Schimitz (1997) trata o cluster como uma aglomeração de empresas gerado de forma espontânea e geograficamente conectados. O autor ainda coloca que existe um ambiente que permite a divisão do trabalho entre as empresas, permitindo-lhes competir além dos seus limites espaciais. Nos países em desenvolvimento, os clusters estão ligados a aspectos culturais o que lhes conduz a um ambiente de confiança e cooperação mútua.

Gonçalves (2000) reforçou a importância de um governo local ativo que proporcione maior infraestrutura e fortaleça a capacidade inovativa das empresas do cluster. O autor ainda aponta que a proximidade geográfica leva as empresas a trocarem ideias, inovações técnicas, colaboração e traz um espírito de coletividade ao ambiente que facilita a comunicação entre elas. O perfil das empresas que participam do cluster é caracterizado por empresas de pequeno e médio porte, desintegração vertical, produção especializada e alta divisão do trabalho.

Os distritos industriais foram designados por Marshall (1920) como uma "concentração de firmas especializadas numa localização particular", sendo considerado uma substituição para o modelo fordista. Ele considerava que empresas com atividades similares geravam benefícios econômicos, posteriormente esta ideia ficou conhecida como "economias externas marshallianas". Tais economias seriam geradas pela alta divisão do trabalho dentro do distrito, criação de infraestrutura, troca de informações, comunicação interna e força de trabalho especializada (KELLER, 2008). 
Ao longo do tempo outros autores ampliaram o conceito de distritos industriais. Cassiolatto e Latres (2003) citaram que a forma como os distritos estão estruturados permite-lhes que economias externas muito significativas sejam geradas, reduzindo os custos e resultando em ganhos de escala, sobretudo para as pequenas empresas. Estudos mais recentes dos distritos focalizam no caso de sucesso da "Terceira Itália" e em outras regiões europeias.

Os principais atributos dos distritos industriais que foram referenciados por Schimitz (1997) são: proximidade geográfica, especialização setorial, predominância de firmas de tamanhos pequeno e médio, colaboração estreita entre as firmas, competição entre as firmas baseada em inovação, uma identidade sociocultural que favorece a confiança, organizações de autoajuda ativas e governos regionais e municipais apoiadores.

Schimitz (1995) alegou que o distrito industrial é sempre um cluster, mas o contrário não é verdadeiro. O Distrito compreende, além de uma concentração setorial e local, uma grande divisão do trabalho em caráter vertical e horizontal entre as empresas. Outro contraste foi colocado por Amin (1996). Este autor caracteriza os clusters como um aglomerado com altos custos, mercado subdesenvolvido para os negócios de serviços e uma cultura familiar no aspecto gerencial. Já os distritos industriais são caracterizados com altos níveis de institucionalização, apoio coletivo, mercado desenvolvido, vantagens pela especialização de tarefas e firmas interdependentes que geram ganhos de associação.

O termo sistema produtivo e inovativo local foi apresentado por Campos e Paula (2006) como um estágio mais avançado dos arranjos produtivos. Conforme os autores apontaram:

[...] o termo arranjos produtivos locais pode ser definido como aglomerações territoriais de agentes econômicos, políticos e sociais, com foco em um conjunto específico de atividades econômicas e que apresentam vínculos e interdependência. Já os sistemas produtivos e inovativos locais são aqueles arranjos produtivos cuja interdependência, articulação e vínculos consistentes resultam em interação, cooperação e aprendizagem, possibilitando inovações de produtos, processos e formatos organizacionais e gerando maior competitividade empresarial e capacitação social. (CAMPOS e PAULA, 2006).

O processo de desenvolvimento local e maturação dos aglomerados foi considerado por Paiva (2002) como um estágio evolutivo, dividido em três etapas. Estas etapas foram chamadas de "momentos marshallianos" do desenvolvimento das aglomerações produtivas. A etapa 1 consistiu na aglomeração das empresas, logo em seguida, esta passa para o estágio de arranjo produtivo. A terceira etapa inicia quando o arranjo passa a coordenar racionalmente seu
A Terceira Itália é caracterizada por aglomerados de pequenas e médias empresas na região nordeste e centro-oeste da Itália. Alto grau de especialização, método de produção flexível e o apoio governamental fizeram desta região um exemplo de desenvolvimento regional. 
desenvolvimento e, assim, transforma-se num sistema local de produção (SLP).

Buscando dar uma definição operacional para um APL, Costa (2011) analisa a evolução de todos os conceitos que envolvem o termo e conclui a importância da aglomeração industrial para o desenvolvimento econômico de uma região devido aos ganhos que a sinergia empresarial gera: atração de mão-de-obra qualificada, inovações tecnológicas, novas empresas para o setor a montante e a jusante, entre outros aspectos. Embora haja discussões conceituais sobre a nomenclatura adequada às aglomerações industriais, como clusters ou Sistemas Locais Produtivos - SLPs, o que não deve mudar é o sentido do objeto de análise (SUZIGAN et al., 2004).

A política brasileira dispõe de regulamentações específicas para a promoção e desenvolvimento dos APLs, como a inclusão do tema nos planos plurianuais PPA 2004-2007 e 2008-2011 e na criação de um grupo de trabalho permanente - GTP APL (MDIC, 2000). Este grupo tem o objetivo de articular as ações governamentais dando apoio integrado aos APLs. Além do grupo, cada estado brasileiro possui um núcleo estadual de apoio aos arranjos produtivos locais.

No Rio Grande do Sul, a política de apoio aos APLs faz parte da "Política Industrial do Estado" dentro do eixo que promove a política de economia da cooperação (SPDI, 2013). Normativamente, esta política está respaldada pelo Decreto $n^{\circ} 48.936$ que regulamenta a Lei $n^{\circ} 13.839$ que institui o "Programa de Fortalecimento das Cadeias e Arranjos Produtivos Locais".

A figura 1 apresenta o mapa do Rio Grande do Sul sendo representado pelos vinte arranjos enquadrados pela AGDI. As áreas coloridas são os municípios que que possuem APLs. As áreas em cinza são os municípios vizinhos que, neste caso, poderiam se beneficiar dos efeitos de transbordamento da política de arranjos. Contudo, tais efeitos não chegaram a serem medidos neste trabalho. A região sul do estado compõe alguns arranjos como o Complexo Industrial da Saúde (APL8), Alimentos da Região Sul (APL6) e Polo Naval de Rio Grande (APL18). A região metropolitana de Porto Alegre apresenta arranjos mais ligados à área tecnológica, máquinas e eletroeletrônica. A maior concentração de APLs está na região da serra gaúcha. Já na região norte e noroeste destacam-se os arranjos da Agroindústria Familiar. 


\section{Figura 1. Arranjos Produtivos Locais no Estado do Rio Grande do Sul.}

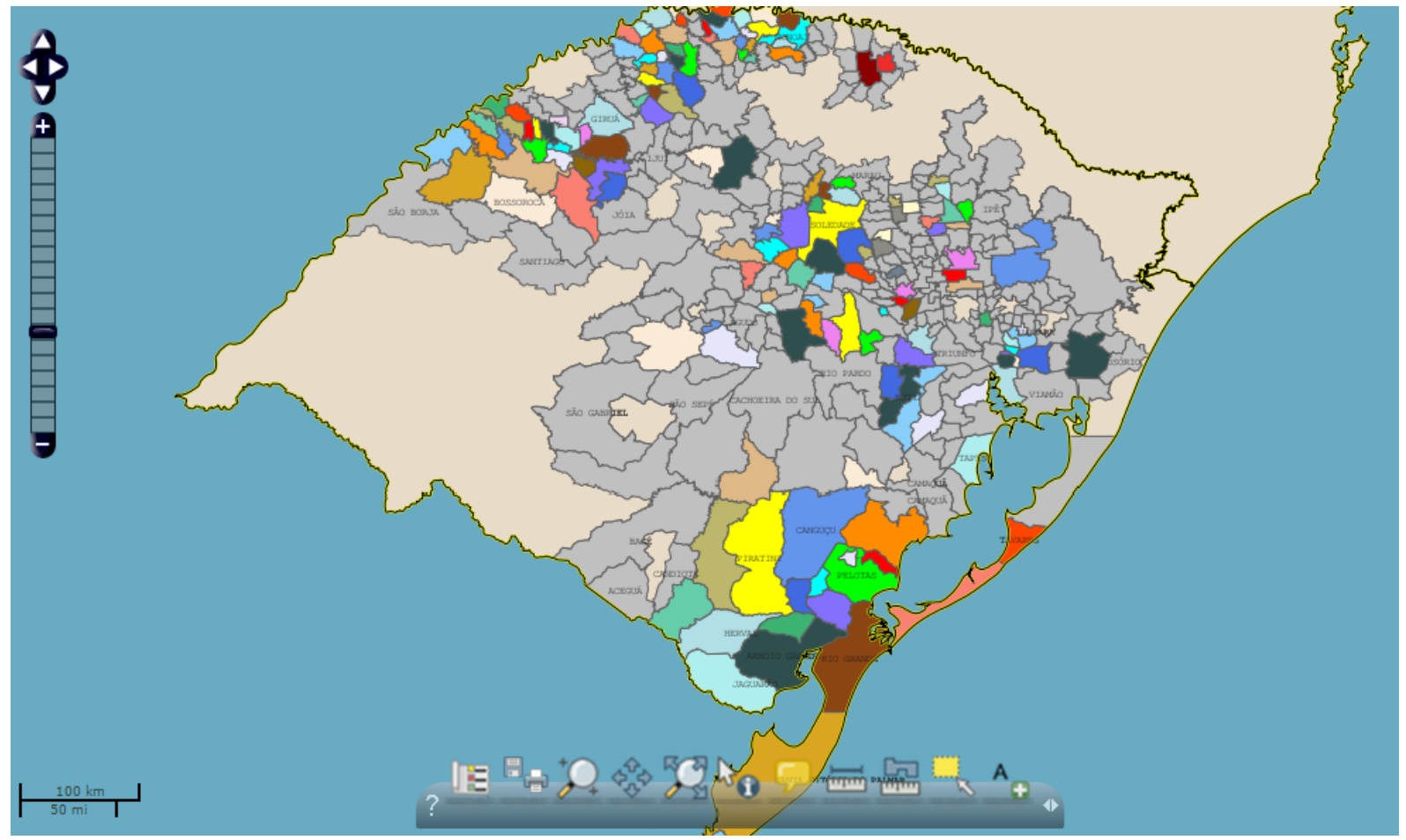

Fonte: i3geo - DIEESE

Entre os APLs existentes no território gaúcho, os casos do setor de Vitivinicultura, de máquinas e implementos agrícolas, metal mecânico e o APL do setor moveleiro são destacados como os mais exitosos (TATSCH e PASSOS, 2008) e são analisados pela literatura. Kirschbaum et al. (2007) estudou as origens e a evolução do arranjo produtivo da Vitivinicultura. Segundo a autora, o APL do vinho refletindo os hábitos dos seus imigrantes, se fortaleceu ao longo do século XX, constituindo vinícolas cooperativas que desenharam inovações que garantiram qualidade ao produto que conquistou mercados no país e fora dele.

Outro exemplo da diversidade de tipologias produtivas que podem ser encontradas em um APL é o arranjo produtivo de máquina e implementos agrícolas localizado no noroeste do Rio Grande do Sul. Tatsch e Passos (2008) elaboram um detalhado estudo sobre este arranjo, destacando as instituições envolvidas e como elas são importantes para o fomento de inovações para soluções para as demandas da fronteira agrícola.

Lançando mão de um questionário para avaliar a relevância de um APL, Dias (2011) aplicou-os aos dirigentes de uma das principais políticas públicas com vistas ao desenvolvimento territorial brasileiro: a "Política Nacional de Desenvolvimento Regional” (PNDR) do “Ministério da Integração Nacional” (MIN). Nessa pesquisa, ele concluiu que os arranjos produtivos locais influenciam positivamente o alcance dos objetivos empresariais e se constituem em uma forte ferramenta de suporte, tendo uma importância de destaque entre os fatores que impulsionam o desenvolvimento.

De forma geral, as metodologias utilizadas para avaliar o desenvolvimento desses arranjos produtivos locais estão baseadas no levantamento de informações por meio da aplicação de um questionário aos participantes do arranjo, procurando captar sua percepção acerca da relevância socioeconômica do mesmo para a região 
onde está inserido, como fazem Dias (2011) e Costa, Andrade e Silva (2006), por exemplo. Uma abordagem complementar é utilizar a perspectiva histórica (vide KIRSCHBAUM et al., 2007) destacando a importância de determinada atividade para o local. Embora sejam abordagens que contribuam para a descrição do processo de implementação e desenvolvimento dos APLs fica registrado a carência de uma abordagem quantitativa que complemente esta análise ou elucide alguma característica não observada anteriormente.

\subsection{Evidências Empíricas de Impacto de uma Política de Aglomeração}

Apesar da experiência internacional em promoção de diferentes políticas de aglomeração de empresas são poucos os trabalhos que avaliam o seu impacto econômico e social. Flack et al. (2010) desenvolveram um trabalho onde buscou-se saber se as políticas locais aumentaram a concorrência entre as firmas neste ambiente. Neste trabalho foi avaliado o impacto de uma política orientada de clusters na Bavária, Alemanha. Essa política selecionou cinco áreas de tecnologia e o seu objetivo foi aumentar a cooperação entre a ciência, negócios e finanças nessas indústrias, com a finalidade de aumentar a aglomeração entre as indústrias locais. $O$ governo gerou incentivos para que essa cooperação existisse, através do programa "The Bavarian High-Tech Offensive", no qual focou na melhoria da infraestrutura pública.

A estratégia empírica adotada por Flack et al. (2010) foi o uso do método de diferenças-em-diferenças (DD). A especificação em DD compara o grau de inovação das firmas bavarianas com firmas de outros estados antes e depois desta política ser introduzida na Bavária. Numa segunda etapa foi avaliado o grau de inovação sem o tratamento do grupo de controle. O que se percebeu foi que, dependo do grau de inovação, o programa aumentou de $4,6 \%$ a $5,7 \%$ a probabilidade das empresas alvo inovarem.

Outro estudo realizado por Martín et al. (2011) analisou empiricamente o programa francês: "Sistemas Produtivos Locais" (SPL). O objetivo do programa é o de aumentar a competitividade entre as empresas. Foram utilizados métodos de diferenças-em-diferenças, tripla diferença e "matching", entre os anos de 1996 a 2004. O que se observou no estudo foi que as firmas adeptas do SPL possuem um fator mais intensivo em trabalho do que aquelas que não participam do programa. Encontrou-se impacto na preservação do nível de emprego, mas não no aumento da produtividade. Martín et al. (Ibid.) também não encontram evidências de impacto sobre o nível de exportações.

Algo muito semelhante foi desenvolvido por Criscuolo et al. (2007) acerca do programa "Assistência Regional Seletiva", na Inglaterra. O programa forneceu subsídios às regiões menos desenvolvidas. Como resultado, elas tiveram um impacto positivo no emprego e investimento, porém, não houve nenhum efeito na produtividade das firmas. Os autores consideraram que tal política pode diminuir a redistribuição de plantas menos eficientes e afetar negativamente o crescimento da produtividade agregada.

Engel et al. (2011) utilizaram o método de diferenças-em-diferenças para avaliar o impacto dos clusters de biotecnologia alemã sobre o grau de pesquisa e desenvolvimento. As medidas de desempenho utilizadas deram-se através do 
número de patentes de biotecnologia e o número de projetos de "Pesquisa e Desenvolvimento" (P\&D) levantados. Os resultados apontaram efeito significativo e positivo no número de projetos públicos em P\&D no período de tratamento. Foi verificado que a política beneficiou as regiões e setores em ascensão. Além disso, não se verificou efeitos de longo prazo pois as firmas "perdedoras" podem ter estabelecido parcerias com as firmas "vencedoras" e, assim, o efeito dissipou-se.

Evidências contrárias à Engel et al. (2011) foram sugeridas por Fontagné et Al. (2013). Eles trouxeram uma abordagem um pouco diferente com relação à avaliação das políticas de clusters na França. Os arranjos foram divididos em três amostras: "universal", "potencialmente universal" e "clusters nacionais", essa metodologia Ihes permitiu contrastar os resultados efetivos do processo de seleção e os objetivos da política de cluster em termos de segmentação. Os autores buscaram evidências na literatura no qual confirma que os formuladores da política escolhem as regiões e setores em "declínio" como alvo de suas ações. Outra hipótese levantada é contrária: são as firmas em declínio que buscam os incentivos governamentais. Este estudo pretendeu avaliar o grau de exportação dos clusters em relação às empresas individuais. Os resultados encontrados, porém, não foram muito satisfatórios: apenas $2 \%$ do total de exportações manufaturadas correspondiam aos clusters.

Para o caso brasileiro, Baré et al. (2012) propuseram avaliar o impacto dos APLs sobre o nível de emprego, exportação e probabilidade de exportação. O estudo foi aplicado para o estado de São Paulo e Minas Gerais, entre os anos de 2002 a 2009. O método utilizado foi diferenças-em-diferenças e também foi aplicado efeito fixo, matching e o método de re-ponderação. Os beneficiários diretos foram as firmas que participam do cluster, os beneficiários indiretos, firmas que não participam do arranjo mas que possuem algum vínculo com os participantes. Já os não beneficiados são as firmas que não participam do APL.

Num primeiro momento os autores tentaram encontrar a relação de efeito causal, que, neste caso, é a diferença entre o valor da variável de interesse antes da política ser aplicada e o valor que teria no caso da ausência da política. Além do grupo de tratamento e controle, também foi necessário avaliar os efeitos de transbordamento (spillovers). O experimento ideal seria a randomização destes grupos, porém este método não foi possível. Para evitar os vieses de seleção (de características observáveis e não observáveis) foi escolhida a estrutura em painel e usou-se o modelo de efeito fixo de regressão linear. Selecionou-se um grupo de controle que fosse similar ao grupo de beneficiários diretos e indiretos, eles também deveriam ter tendências de pré-tratamento muito próximas. Como forma de reforçar a estimação usou-se o balanceamento entrópico e um método multivariado de reponderação. Este último permitiu reponderar toda a amostra de tal modo que o grupo de controle coincidiu com as variáveis independentes do grupo de tratamento.

Os dados do CNPJ das empresas participantes do APL foram pareados com as informações contidas na RAIS e na Secretaria do Comércio Exterior do Brasil, além disso usou-se a base de dados das firmas registradas no SEBRAE. Com o estudo, observou-se que a política de APL, através das externalidades do mercado de trabalho, pode fomentar a criação dos polos especializados que permitiu que as firmas desse cluster demandassem mais emprego para outras áreas com fins parecidos. 
Foi encontrado um efeito médio positivo e direto no emprego: o programa causou um aumento de dez trabalhadores por beneficiário direto. Em economias de aglomeração, o mercado de trabalho; o conhecimento tácito e os fornecedores são externalidades diretas que geralmente melhoram a produtividade total dos fatores e o desempenho das empresas. Além disso, o ambiente de aglomeração pode atrair novas firmas ou aumentar as já existentes. A transmissão de conhecimento entre os colaboradores do cluster permite que em momentos de crise seja possível realoca-los de forma mais eficiente. Por outro lado, o aumento de trabalhadores pode ser fruto da realocação dos beneficiários indiretos para os diretos.

Para os níveis de exportações o impacto foi grande e significativo, mas decresceu ao longo do tempo. O grupo de controle não foi suficientemente bom para este nível de comparação, o que acabou gerando uma dificuldade para os autores no momento de delinear conclusões mais precisas. O efeito para a variável probabilidade de exportação foi altamente significativo e causou um aumento de quatro a cinco por cento para os beneficiários diretos, já para os beneficiários indiretos esse valor ficou abaixo de um por cento. De uma forma geral, observou-se um efeito de transbordamento positivo sobre os vizinhos do cluster.

A estratégia de pesquisa apresentada a seguir busca contribuir para a literatura avaliando a política dos APLs apoiados no estado do Rio Grande do Sul. O foco da avaliação está sobre o nível de emprego e salário médio do setor.

\section{Metodologia}

Atualmente, existem 34 arranjos identificados pelo Observatório Nacional de APLs, mas apenas 20 destes são apoiados pela Agência Gaúcha de Desenvolvimento e Promoção do Investimento (AGDI). Desse modo, apenas os arranjos apoiados pela AGDI fizeram parte da análise.

As informações referentes ao nível de emprego e salário médio nominal foram retiradas do site da Relação Anual de Informações Sociais (RAIS). O nível de desagregação das variáveis está estruturado no nível de classes CNAE (Classificação Nacional de Atividades Econômicas), correspondendo a um total de 673 classes.

O período estudado foi de 2006 a 2014. Entre 2004 e 2006 a CNAE 1.0 foi reestruturada e se transformou em CNAE 2.0, algumas classes foram adicionadas à classificação enquanto que outras foram extinguidas e reintegradas a outras categorias. Assim, não foi possível utilizar os dados anteriores a 2005 pois o número de classes reestruturadas foi significativo e poderia comprometer a eficiência da análise.

O método utilizado foi o de diferenças-em-diferenças (DD). O método consiste, num primeiro momento, na diferença das médias da variável de análise antes e depois do programa, tanto para o grupo de tratamento quanto para o de controle. Num segundo momento, calcula-se a diferença da primeira diferença já calculada. A principal hipótese desse modelo é que a trajetória temporal da variável em análise do grupo de controle representa o que ocorreria com o grupo tratado caso não tivesse ocorrido a intervenção. Supõe-se que a trajetória das duas são semelhantes antes do programa e, após o programa, a variável tratada apresentará diferença de trajetória da variável controle. 
O grupo tratado são os setores beneficiados pelo APL e grupo de controle os não beneficiados. Os municípios do grupo de controle devem possuir características semelhantes aos municípios que possuem APLs. Ou seja, supõe-se que na ausência do programa, a variável de tratamento não sofreria alterações na sua trajetória. Logo, pretende-se avaliar se o fato dos setores dos municípios que receberam o tratamento (possuem APLs) tiveram resultados acima dos setores do grupo de controle em relação ao número de empregos gerados e o salário médio para o período estudado.

A comparação foi feita entre os beneficiários diretos, ou seja, os setores dos municípios que possuem APL, e os não beneficiados. Essa comparação dá impacto direto da política:

$$
\mathrm{EDT}=\mathrm{E}[\mathrm{Yi} \mid \mathrm{Di}=1, \mathrm{Ci}=1]-\mathrm{E}[\mathrm{Yi} \mid \mathrm{Di}=0, \mathrm{Ci}=0]
$$

$\mathrm{EDT}=$ efeito direto do tratamento

$\mathrm{Yi}=1$ : produto das firmas que participam do APL

$\mathrm{Di}=1$ : municípios que são recebem o tratamento

$\mathrm{Ci}=1$ : setores que participam do APL

$\mathrm{Yi}=0$ : produto das firmas que não participam do APL

$\mathrm{Di}=0$ : municípios do grupo controle

$\mathrm{Ci}=0$ : setores que não participam do APL

Porém, é razoável supor que haveria diferença na produção entre os beneficiários e não beneficiários mesmo na ausência da política. Ou seja, eles já possuíam características distintas mesmo antes do tratamento. Portanto, uma comparação entre a média de produto entre esses dois grupos poderia estimar de forma viesada o efeito da política.

Existem variáveis não observáveis, como o comportamento empreendedor ou o grau de inovação e governança, que variam entre as empresas e são constantes ao longo do tempo. Logo, o efeito fixo nos dados em painel é uma forma interessante de eliminar essa incerteza entre os dados observáveis e não observáveis. O efeito foi aplicado através de variáveis dummies para cada setor CNAE dos municípios. Além disso, acredita-se que outras políticas do governo e acordos podem ter influenciado os arranjos ao longo do tempo. Como forma de ajuste, aplicou-se variáveis dummies de tempo para os anos de 2006 a 2014. Logo, o modelo que está sendo tratado é chamado de efeitos fixos bidirecionais (CAMERON e TRIVEDI, 2005).

A hipótese trazida pelo modelo de efeito fixo, em que a trajetória do grupo de tratamento seguiria a mesma trajetória do grupo de controle na ausência da política, pode ser contestável. Poderia supor-se que os setores a serem comparados entre os municípios são heterogêneos. Logo, a fim de reforçar os pressupostos de identificação, foi aplicado o método de pareamento "Propensity Score Matching". O grupo de controle foi comparado ao grupo de tratamento com base numa série de características observáveis. $O$ escore de propensão $P(x)$ é definido como a probabilidade condicional de um indivíduo receber o tratamento dado suas características observáveis X (RESENDE e OLIVEIRA, 2008). 
Quadro 1. Método de diferenças-em-diferenças

\begin{tabular}{|c|c|c|}
\hline $\begin{array}{c}\text { Antes do início do } \\
\text { programa }\end{array}$ & Grupo de Tratamento & Grupo de Controle \\
\hline $\begin{array}{c}\text { Depois do início do } \\
\text { programa }\end{array}$ & $Y_{i 0}^{T}=\alpha+\beta$ & $Y_{i 0}^{c}=\alpha$ \\
\hline Diferença & $\Delta_{i}^{T}=Y_{i 1}^{T}-Y_{i 0}^{T}=\gamma+\delta$ & $Y_{i 1}^{c}=\alpha+\gamma$ \\
\hline & $\Delta_{i}^{c}=Y_{i 1}^{c}-Y_{i 0}^{c}=\gamma$ \\
\hline Diff-in-diff & $Y_{i}^{T}=\alpha+\beta T_{i}+\gamma t_{i}+\delta\left(T_{i} * t_{i}\right)+\varepsilon_{i}$ \\
\hline
\end{tabular}

Fonte: Elaborado pela autora.

O modelo é apresentado da seguinte maneira:

$$
Y_{i}=\alpha+\beta T_{i}+\gamma t_{i}+\delta\left(T_{i} * t_{i}\right)+\varepsilon_{i}
$$

"Yi" representa o impacto sobre o salário médio nominal do setor caso "i" seja igual a um e número de vínculos ativos para "i" igual a zero, " $\alpha$ " é o valor esperado da variável estudada quando se analisa o grupo de controle antes da mudança. " $\beta$ " reflete o impacto do grupo de tratamento na variável estudada (diferença permanente entre controle e tratamento). " $\gamma$ " é o impacto do segundo período sobre a variável estudada (tendência no tempo comum entre controle e tratamento). "Ti" representa a variável dummy. 1 para o grupo de tratamento e o para o grupo de controle e "ti" é outra variável dummy. 1 momento pós-política e o para o período pré-política. " $\delta$ " dá o impacto pós-evento do grupo de tratamento, em relação ao grupo de controle, sobre a variável estudada (efeito verdadeiro do tratamento), " $\varepsilon_{i}$ " é o termo de erro aleatório não observado.

Como o fato de um município ter ou não ter um APL apoiado em sua região geográfica não é um evento aleatório, o modelo de efeito fixo captura as características não observáveis dos municípios que podem explicar o fato do município ter ou não um APL apoiado (questões de capital social, estrutura política, organização produtiva já existente, etc...) que são fixas no tempo. Sob essa condição o modelo de efeito fixo gera um estimador consistente para avaliar o efeito médio da política pública de auxílio aos setores tratados.

\section{RESULTADOS ESTIMADOS}

A política de APLs no Rio Grande do Sul iniciou sua trajetória em 1999, um pouco antes das ações terem ganho âmbito nacional. Ao longo do tempo, a política de arranjos no estado pode ser dividida em quatro fases distintas que coincidem com as trocas de governo (AGDI, 2011): a primeira gestão foi de 1999 até 2002 com o "Programa de Apoio aos Sistemas Locais de Produção"; a segunda ocorreu no 
período de 2003 a 2006 com a denominação "Programa para Apoio aos Arranjos Produtivos Locais"; a terceira aconteceu entre 2007 a 2010 com a mesma denominação; e, a partir de 2011, o governo lançou uma parceria com o Banco Mundial iniciando o projeto APL, tendo a AGDI como principal gestora.

Como mostra a tabela 1, os APLs foram criados ao longo do tempo. Em 1999 foram criados os dois primeiros APLs do estado: o APL de alimentos da região sul e o moveleiro da serra gaúcha. Entre 1999 a 2011 apenas seis foram enquadrados, isso se deve às fases de volatilidade governamental e às mudanças estruturais dos arranjos. Após a parceria com o Banco Mundial em 2011, criaram-se leis e regulamentações para o apoio aos APLs, resultando também na criação de doze novos arranjos entre 2012 e 2013.

Tabela 1 - Número de APLs por data de formação

\begin{tabular}{c|c}
\hline Ano & APLs \\
\hline 1999 & 2 \\
2003 & 2 \\
2004 & 1 \\
2006 & 1 \\
2008 & 1 \\
2011 & 1 \\
2012 & 6 \\
2013 & 6 \\
\hline Total & 20 \\
\hline
\end{tabular}

Fonte: Elaborado pela autora.

Neste trabalho os vinte APLs foram agrupados em ordem alfabética, conforme mostra a tabela 2. Embora alguns arranjos tenham nomenclaturas parecidas, cada um deles é formado por diferentes grupos de setores. O arranjo Audiovisual, por exemplo, compreende setores como: atividades de produção cinematográfica, de vídeos e de programas de televisão, distribuição cinematográfica, atividades de gravação de som e de edição de música, programadoras e atividades relacionadas à televisão por assinatura, atividades pósprodução cinematográficas, atividades de exibição cinematográfica e atividades de televisão aberta. 
Tabela 2. Nomenclatura dos APLs Apoiados pela AGDI Avaliados:

\begin{tabular}{l|c}
\hline APL 1 & Agroindústria Familiar Da Região Celeiro \\
\hline APL 2 & Agroindústria Familiar Da Região Missões \\
\hline APL 3 & Agroindústria Familiar Da Região Médio Alto Uruguai \\
\hline APL 4 & Agroindústria Familiar Da Região Do Vale Do Taquari \\
\hline APL 5 & Agroindústria Familiar Da Região Vale Do Rio Pardo \\
\hline APL 6 & Alimentos da Região Sul \\
\hline APL 7 & Audiovisual \\
\hline APL 8 & Complexo Industrial Da Saúde \\
\hline APL 9 & Metroeletônico De Automação E Controle \\
\hline APL10 & Metalmecânico E Automotivo Da Região Central \\
\hline APL11 & Metalmecânico Pós-colheita \\
\hline APL12 & Moveleiro Da Serra Gaúcha \\
\hline APL13 & Máquinas e Equipamentos Industriais \\
\hline APL14 & Pedras, Gemas E Joias \\
\hline APL15 & Polo De Moda Da Serra Gaúcha \\
\hline APL16 & Polo Naval do Jacuí \\
\hline APL17 & Polo Naval E Offshore De Rio Grande E Entorno \\
\hline APL18 & Tecnologia Da Informação Da Serra Gaúcha \\
\hline APL19 & Tecnologia Da Informação E Comunicação Da Região Central \\
\hline APL20 &
\end{tabular}

Fonte: elaborado pela autora.

A tabela 3 apresenta os resultados estimados para o nível de emprego. Houve um impacto positivo e significativo sobre dois dos vinte APLs estudados. Isso significa que os setores identificados no APL Moveleiro da Serra Gaúcha tiveram (APL13), na média, 89 vínculos empregatícios a mais que os setores que não possuem a política. O APL de Gemas e Joias (APL15) apresentou um aumento médio de 4 vínculos ativos se comparado aos setores que não possuem APL. Comparando-se aos resultados do artigo de Baré et al. (2012), esperava-se encontrar mais efeitos significativos para esta variável no trabalho apresentado. Pelos resultados expostos em seu artigo, todos os clusters tiveram efeitos positivos no nível de emprego, variando de cinco a dezesseis empregados a mais em relação às empresas que não possuem a política. 
Tabela 3. Resultado para o modelo - número de vínculos ativos:

\begin{tabular}{ccccc}
\hline & Coef & Std. Err & $T$ & P>t \\
\hline APL_1 & -27.15 & 9.29 & -2.92 & 0.004 \\
APL_2 & 2.41 & 3.22 & 0.75 & 0.454 \\
APL_3 & -14.12 & 6.55 & -2.15 & 0.032 \\
APL_4 & 3.63 & 3.78 & 0.96 & 0.338 \\
APL_5 & -7.08 & 4.03 & -1.76 & 0.079 \\
APL_6 & 15.58 & 13.18 & 1.18 & 0.238 \\
APL_7 & -207.66 & 87.68 & -2.37 & 0.018 \\
APL_8 & 36.61 & 21.35 & 1.71 & 0.087 \\
APL_9 & 174.64 & 112.82 & 1.55 & 0.122 \\
APL_10 & -15.87 & 8.36 & -1.90 & 0.058 \\
APL_11 & 30.48 & 19.01 & 1.60 & 0.110 \\
APL_12 & 128.44 & 106.71 & 1.20 & 0.229 \\
APL_13 & 89.52 & 40.31 & 2.22 & 0.027 \\
APL_14 & -54.88 & 57.71 & -0.95 & 0.342 \\
APL_15 & 4.46 & 2.17 & 2.06 & 0.040 \\
APL_16 & 24.64 & 13.08 & 1.88 & 0.060 \\
APL_17 & 9.70 & 5.35 & 1.81 & 0.070 \\
APL_18 & 17.27 & 31.60 & 0.55 & 0.585 \\
APL_19 & -6.46 & 4.72 & -1.37 & 0.172 \\
APL_20 & -6.13 & 5.42 & -1.13 & 0.259 \\
Observações & 3.035 .916 & & & \\
R & 0,158 & & & \\
\hline
\end{tabular}

Fonte: elaborado pela autora.

Nota: os realces em cinza representam os resultados significativos e positivos.

O governo do estado tem apoiado o APL Moveleiro desde a sua formação em 1999, porém, a origem da indústria moveleira nesta região está relacionada à chegada dos imigrantes italianos no século XIX. Além de ser um APL tradicional no estado, ele também é o segundo maior produtor de móveis do Brasil (MACADAR, 2007). Assim, o resultado encontrado para este APL confirma seu estágio avançado de desenvolvimento.

O APL da Agroindústria Familiar da Região Celeiro, Agroindústria Familiar da Região Alto Médio Uruguai e Audiovisual tiveram efeito significativo, porém o impacto foi negativo. Os dois arranjos ( $A P L 1$ e 3 ) ligados a agroindústria familiar foram criados em 2012 e o arranjo Audiovisual (APL7) foi criado em 2013, assim, o tempo de exposição de tratamento foi muito curto. Vale ainda destacar que a dificuldade de encontrar co-variadas para os níveis setoriais pode ter contribuído para tais resultados não esperados.

É importante frisar que $60 \%$ dos APLs foram constituídos a partir de 2012, ou seja, a análise de impacto de dois anos da implantação da política pode não ser o tempo suficiente para que se possa observar diferenças entre o grupo tratado e o grupo controle.

É importante destacar dois limites da abordagem adotada que pode estar associada ao resultado obtido. Primeiro, a abordagem setor e ano com efeito fixo corrige os efeitos dinâmicos dos setores, porém não foi possível controlar os mesmos 
efeitos em nível de firma. Logo, os efeitos da política poderiam ter sido notados em firmas específicas que tornam os valores insignificantes para o setor. Segundo, alguns segmentos não possuem um contra factual passível de comparações. Um exemplo disto é o APL audiovisual (APL7) que abrange a região metropolitana de Porto Alegre. Estes municípios centralizam quase 70\% dos estabelecimentos e agregam 80,1\% dos trabalhadores do setor audiovisual no estado (SCHIAVO, 2015). Outro exemplo a ser ressaltado é o Complexo Industrial da Saúde (APL9) que compreende a cidade de Pelotas e seu entorno. Portanto, é possível que para alguns setores o grupo de controle não seja o melhor grupo de comparação.

A tabela 4 apresenta os resultados obtidos para a variável renda média nominal no qual metade dos APLs apresentaram resultados significativos. O único que apresentou significância, mas com resultado negativo foi o arranjo da Agroindústria Familiar da Região do Vale do Rio Pardo (APL5).

Os APLs da região sul do estado apresentaram resultados significativos. Em comparação aos mesmos setores, a remuneração média nominal do APL da saúde (APL8) é R\$272,00 a mais que o grupo controle. O APL Naval de Rio Grande (APL18) possui uma renda média de $\mathrm{R} \$ 181,00$ a mais se comparado com os mesmos setores que não possuem a política.

Tabela 4. Resultado para modelo - salário médio nominal

\begin{tabular}{ccccc}
\hline & Coef & Std. Err & $t$ & $P>t$ \\
\hline APL_1 & -33.37 & 20.59 & -1.62 & 0.106 \\
APL_2 & 3.96 & 8.57 & 0.46 & 0.644 \\
APL_3 & -1.42 & 17.04 & -0.08 & 0.933 \\
APL_4 & -0.15 & 6.47 & -0.02 & 0.981 \\
APL_5 & -34.41 & 10.09 & -3.41 & 0.001 \\
APL_6 & 38.23 & 18.61 & 2.05 & 0.040 \\
APL_7 & -52.11 & 46.69 & -1.12 & 0.265 \\
APL_8 & 272.32 & 55.08 & 4.94 & 0.000 \\
APL_9 & 354.91 & 73.67 & 4.82 & 0.000 \\
APL_10 & 56.77 & 64.45 & 0.88 & 0.379 \\
APL_11 & 19.49 & 11.26 & 1.73 & 0.084 \\
APL_12 & 302.33 & 135.21 & 2.24 & 0.026 \\
APL_13 & 130.34 & 26.11 & 4.99 & 0.000 \\
APL_14 & 175.89 & 42.15 & 4.17 & 0.000 \\
APL_15 & 49.22 & 15.29 & 3.22 & 0.001 \\
APL_16 & 50.15 & 15.99 & 3.14 & 0.002 \\
APL_17 & 26.62 & 37.56 & 0.71 & 0.479 \\
APL_18 & 181.87 & 68.74 & 2.65 & 0.008 \\
APL_19 & 32.40 & 35.96 & 0.90 & 0.368 \\
APL_20 & 122.54 & 115.03 & 1.07 & 0.287 \\
Observações & 3.035 .916 & & & \\
R & 0,286 & & & \\
\hline Fond & & & &
\end{tabular}

Fonte: elaborado pela autora.

* Nota: os realces em cinza representam os resultados significativos e positivos. 
A região da serra e metropolitana de Porto Alegre também tiveram resultados interessantes. Os trabalhadores do Arranjo de Automação e Controle (APL9) ganharam, em média, $\mathrm{R} \$ 354,91$ a mais que os mesmos setores do grupo de controle. O arranjo moveleiro da serra (APL13) e o de máquinas e equipamentos industriais (APL14) tiveram resultados expressivos: $R \$ 130,35$ e $R \$ 175,89$, respectivamente.

Um estudo feito por Carvalho et al. (2015) constatou que APL Metalmecânico Pós-Colheita (APL12) foi o único que teve um efeito positivo e significativo em relação ao PIB per capita se comparado aos municípios que não possuem APL. Complementando este estudo pode-se observar que o salário médio dos trabalhadores que fazem parte deste APL é de 302 reais a mais se comparado aos demais setores que não possuem a política.

Observou-se que nenhum dos APLs ligados a agroindústria familiar, com exceção do Vale do Rio Pardo (APL5), obtiveram resultados com significância. Isso significa que estes arranjos não apresentaram diferenças salariais se comparados ao grupo de controle. O setor do agronegócio pode afetar outros setores, como o de comércio e serviços (FEIX, 2015) e, assim, há a possibilidade dos arranjos estarem impactando em setores que sejam os setores base identificados no APL.

Outro ponto percebido foi que, no geral, os setores agroindustriais não atingiram resultados tão relevantes quanto os setores industriais. Para Vian et al. (2013) os mercados não são homogêneos, cada um possui estrutura e dinâmicas diferentes. O progresso tecnológico e as inovações são fatores decisivos para o setor permanecer no mercado. Desse modo, seria possível dizer que os arranjos com resultados favoráveis possuem uma estrutura que facilita a formação de clusters numa governança mais avançada.

Como mencionado anteriormente, o apoio aos arranjos ficou enfraquecido e até mesmo chegou a ser cancelado no período de trocas de governo. Durante a gestão de 2007 a 2010 o governo passou a ser um coadjuvante na promoção do desenvolvimento econômico e regional devido à redução de gastos (AGDI, 2011). Tais acontecimentos podem ter afetado negativamente o desempenho dos arranjos por conta desta descontinuidade de incentivos.

Em contrapartida, o governo criou em 2009 a "Lei da Inovação do Estado do Rio Grande do Sul" (Lei na 13.196 de 13/07/2009) que prevê o apoio e consolidação dos APLs através da incorporação de novas tecnologias. Além disso, em 2011 o governo do estado fechou um contrato com o Banco Mundial com intuito de apoiar vários projetos, inclusive os arranjos. Assim, a partir de 2011 uma estrutura mais eficiente foi consolidada que contou com a criação de leis, regulamentos e aumento da equipe técnica a fim de garantir a continuidade do suporte aos APLs. Outro projeto, o Fundo APL, criado pela Lei 13.840 de 05/12/2012 tem como objetivo disponibilizar recursos financeiros aos cooperados dos arranjos. Isso demostra que, apesar da existência dos APLs ser antiga, a sua fase de maturação foi, de fato, iniciada em 2012. Por consequência é possível que os resultados não significativos de alguns arranjos tenham sido em função deste pouco tempo e exposição ao efeito do tratamento (de 2011/2012 a 2014). 


\section{CONCLUSÃO}

Nas últimas décadas tem crescido o debate político sobre o apoio a arranjos produtivos locais como instrumento de política pública de promoção ao desenvolvimento regional. Apesar do avanço político desse instrumento pouco ainda se tem de avaliação de seu impacto sobre o desempenho econômico. Esse artigo objetivou contribuir para a essa literatura oferecendo uma tentativa de avaliação do efeito causal do apoio aos APLs sobre as variáveis emprego e remuneração dos setores apoiados por APLs.

No geral, os resultados apontaram um efeito positivo e significativo sobre o salário médio em doze dos vinte APLs. O arranjo Eletroeletrônico de Automação e Controle e o APL Metalmecânico pós-colheita foram os que apresentaram os maiores aumentos. Já para a variável nível de vínculos empregatícios o efeito foi menor, o APL Moveleiro da Serra Gaúcha e o arranjo de Pedras, Gemas e Joias foram os únicos que alcançaram o objetivo de promover o emprego nos municípios que foram atingidos pela política. Estes resultados são iniciais e podem refletir o fato de muitos setores estarem a pouco tempo participando de uma política de apoio.

Outro ponto muito importante a se destacar está relacionado às limitações do trabalho. Num primeiro momento pretendia-se analisar o impacto em nível de firma para capturar com maior precisão quais empresas fazem parte do APL. Como não foi possível ter acesso ao CNPJ destas empresas, a seleção foi feita por setores CNAE 2.0 para cada município. A análise sobre o nível de exportações teve que ficar de fora por conta do grande número de setores que seria necessário converter (código NCM em CNAE). Além disso, corria-se o risco de haver sub ou sobrestimação dos resultados por conta desta conversão.

Para melhor avaliar a política pública de incentivo aos APLs, este artigo abordou o seu impacto em termos setoriais. É possível que o impacto da política de apoio aos APLs esteja no nível da firma. Tal fato pode ser um limitante do presente trabalho, por isto postula-se que novos trabalhos possam avaliar o impacto sobre a renda e emprego utilizando micro dados a nível de firma, contribuindo para a presente discussão e debate.

\section{REFERÊNCIAS}

AGDI, Agência Gaúcha de Desenvolvimento e Promoção do Investimento. Programa de Apoio à Retomada do Desenvolvimento do Estado do Rio Grande do Sul PROREDES-BIRD, 2011. Disponível em:

http://www.agdi.rs.gov.br/upload/1351175225_Projeto\%20BIRD\%20APLs\%20$\% 20 H i s t \% C 3 \%$ Bzrico.pdf

AMIN, Asfi. The difference between small firm clusters and industrial districts. In: Seminário Internacional sobre Políticas Industriais Descentralizadas. Brasília, 1996.

BARÉ, Gonzalo Vázquez. DENEGRI, João Alberto. GARONE, Lucas Figal. MAFFIOLI, Alessandro. RODRIGUEZ, Cesar M. Assessing the Impact of Cluster Policies: the case of the Arranjos Productivos Locais in Brazil. Inter-American Development Bank: 2012. 
BINDER, Marcelo Pereira. BRITO, Luiz Arthur Ledur. CARVALHO, Luiz Felipe Nasser. KIRSCHBAUM, Charles. VASCONCELOS, Flávio Carvalho de. Os desafios do cluster vitivinícola da serra gaúcha. In: Serio, Luiz Carlos Di. (Organizador). Clusters empresariais no Brasil: casos selecionados. São Paulo: Saraiva, 2007, 194p.

BOURLEGAT, Cleonice Alexandre Le. Princípios de organização e desempenho de um modelo econômico agroindustrial emergente no centro-oeste: o caso do arranjo produtivo local da fécula de mandioca. In: LASTRES, Helena M. M.; CASSIOLATO, José E. (Orgs.). Estratégias para o desenvolvimento: um enfoque sobre arranjos produtivos locais do Norte, Nordeste e Centro-oeste brasileiros. Rio de Janeiro: Epapers, 2006, 288p.

BRITTO, Jorge. ALBAGLI, Sarita. Glossário de Arranjos e Sistemas Produtivos e Inovativos Locais. Rede de Pesquisa em Sistemas Produtivos e Inovativos Locais. (REDESIST), Rio de Janeiro, 2003.

CAMERON AC, Trivedi PK. Microeconometrics Methods and Applications. Cambridge: Cambridge University Press; 2005.

CAMPOS, Antonio Carlos de; PAULA, Nilson Maciel de. Novas formas de organização industrial e o conceito de firma: uma abordagem neo-schumpeteriana. Ensaios FEE, Porto Alegre, v.27, n.1, 2006.

CAMPOS, Renato Ramos. MATOS, Marcelo. STALLIVIERI, Fabio . VARGAS, Marco Antônio. Políticas Estaduais para Arranjos Produtivos Locais no Sul, Sudeste e Centro-Oeste do Brasil. Rio de Janeiro: E-papers, 2010.

CARVALHO, Ferreira David. SANTANA, Antônio Cordeiro de. MENDES, Fernando A. Teixeira. Análise de Cluster da indústria de móveis de madeira do Pará. Novos Cadernos do NAEA, V.9, N.2, Dez/2006.

CASSIOLATO, José Eduardo; LASTRES, Helena M. M. O foco em arranjos produtivos e inovativos locais de micro e pequenas empresas. In: Lastres, H.M.M; Cassiolato, J.E.e Maciel, M.L. (orgs). Pequena empresa: cooperação e desenvolvimento local. Relume Dumará Editora, Rio de Janeiro, 2003.

CASTRO, José Márcio de. VALE, Gláucia Maria Vasconcellos. Arranjos Produtivos Locais, Distritos Industriais: Reflexões sobre Aglomerações Produtivas. Revista Análise Econômica, p. 81-97, 2010.

COSTA, Francisco de Assis. ANDRADE, Wanderlino Demetrio Castro de. SILVA, Paulo Cunha Fiock da. O Processamento de Frutas no Nordeste Paraense e Região Metropolitana de Belém - Um Arranjo Produtivo Emergente. Rio de Janeiro: Rede Sist (relatório de pesquisa), 2004. 
CRISCUOLO, Chiara. MARTIN, Ralf. OVERMAN, Henry. VAN REENEN, John. The Effect of Industrial Policy on Corporate Performance: Evidence from Panel Data, Paper para discussão, 2007.

DIAS, Cleidson. Arranjos produtivos locais como estratégia de desenvolvimento. Desenvolvimento em questão. Editora Unijuí. ano 9 • n. 17 . jan/jun. 2011.

ENGEL, Dirk. MITZE, Timo. PATUELLI, Roberto. REINKOWSKI, Jamina. Does Cluster Policy Trigger R\&D Activity? Evidence from German Biotech Contests. Rimini Centre for Economic Analysis Working Paper, WP 11-15. 2011.

FALCK, Oliver. HEBLICH, Stephan. KIPAR, Stefan. Industrial Innovation: Direct Evidence From a Cluster-oriented Policy. Regional Science and Urban Economics. Alemanha: Elsevier, 2010.

FEIX, Rodrigo Daniel. LEUSIN, Sérgio Júnior. Painel do Agronegócio no Rio Grande do Sul - 2015. Fundação de Economia e Estatística. Porto Alegre, 2015. Disponível em: http://www.fee.rs.gov.br/wp-content/uploads/2015/09/20150903painel-doagronegocio-no-rs-2015.pdf

FOGUEL, Miguel Nathan. Avaliação Econômica de Projetos Sociais. Fundação Itaú Social. São Paulo: Dinâmica Gráfica e Editora, 2012.

FONTAGNE, Lionel. KOENIG, Pamina. MAYNERIS, Florian. PONCET, Sandra. Cluster Policies and Firm Selection Evidences from France. Journal of Regional Science, p. 126, 2013.

GALDINO, Afonso Carlos. GARCIA, Luís Alberto Ferreira. A Estrutura, Conduta e Desempenho da Indústria Brasileira de Cimento a partir 1990. Cascavel, 2008. VII Seminário do Centro de Ciências Sociais Aplicadas Campus Cascavel. Junho, 2008.

GASTALDON, Murialdo Canto. O Segmento Plástico no Sul Catarinense: uma abordagem sobre a situação recente à luz da problemática dos clusters e distritos industriais. 2000. 237 p. Dissertação de mestrado. Programa de Pós-Graduação em Economia. Universidade Federal de Santa Catarina. Florianópolis, 2000. Disponível em:

https://repositorio.ufsc.br/xmlui/bitstream/handle/123456789/78408/172860.pdf?seq uence=1\&isAllowed $=y$

GONÇALVES, José Correia. Avaliação do Centro Tecnológico Moveleiro no "Cluster" Industrial de Móveis da Região de São Bento do Sul. Dissertação de Mestrado - Centro Sócio-Econômico, Programa de Pós-Graduação em Economia Universidade Federal de Santa Catarina, Florianópolis, 2000. Disponível em: https://repositorio.ufsc.br/handle/123456789/111367 
INMTRO - Instituto Nacional de Metrologia, Qualidade e Tecnologia. O INMETRO e os Arranjos Produtivos Locais. Disponível em:

http://www.inmetro.gov.br/apls/apresentacao.asp. Acessado em: 15 jan. 2015.

KELLER, Paulo Fernandes. Clusters, distritos industriais e cooperação interfirmas: uma revisão da literatura. Revista Economia \& Gestão - PUC Minas, v.8, nº16 janeiro-abril, 2008.

KIRSCHBAUM, Charles; CARVALHO, Luiz Felipe Nasser; BRITO, Luiz Arthur Ledur; BINDER, Marcelo Pereira; VASCONCELOS, Flávio Carvalho de. Os desafios do cluster vitivinícola da serra gaúcha. In: Serio, Luiz Carlos Di. (Organizador). Clusters empresariais no Brasil: casos selecionados. São Paulo: Saraiva, 2007.

KUPFER, David. HASENCLEVER, Lia. Economia Industrial: Fundamentos teóricos e práticas no Brasil. Rio de Janeiro, 2002.

LIGO, Alexandre K. FONSECA, Luis Paulo B. SOUZA, Marco Antonio de. YAZAKI, Paulo Akihumi. FASTI, Ricardo. O sol brilha para o cluster de moda praia. In: Serio, Luiz Carlos Di. (Organizador). Clusters empresariais no Brasil: casos selecionados. São Paulo: Saraiva, 2007.

MARITN, P., Mayer, T., MAYNERIS, F. Public support to clusters: a firm level study of French 'Local Productive Systems'. Regional Science and Urban Economics, p. 108123, 2011.

MARSHALL, Alfred. Principle of economics. 8. Ed. Macmilan, 1920. Ministério do Desenvolvimento, Indústria e Comércio Exterior. Políticas Públicas. Disponível em: http://portalapl.ibict.br/menu/itens_menu/apls/politicas_publicas_APL.html. Acessado em: 25 mar. 2015.

Núcleo Estadual de Arranjos Produtivos Locais do Espírito Santo. Termo de Referência Para Atuação do Núcleo Estadual de APL do Espírito Santo (NE APL/ES). Disponível em

http://www.sedes.es.gov.br/images/arquivos/downloads/1._termo_de_referncia_pa ra_atuao_do_ne-apl_es.pdf. Acessado em 12 jan. 2015.

PAIVA, Carlos Águedo. O que são Sistemas Locais de Produção (e por que eles são tão importantes na estratégia de desenvolvimento do Governo Democrático e Popular no Rio Grande do Sul). In: Encontro de Economia Gaúcha, v.1, 2002, Porto Alegre, Anais... (CD-ROM)

PORTER, Michael E. A Vantagem Competitiva das Nações. Rio de janeiro, Campus, 1993.

PORTER, Michael. Clusters and the new economics of competition. Harvard Business Review. England, v.6, n.10, 1998. 
POSSAS, Mário Luiz. Estruturas de Mercado em Oligopólio. São Paulo: Editora Hucitec, 1990.

POSSAS, Silvia. Concorrência e competitividade: notas sobre estratégia e dinâmica seletiva na economia capitalista. São Paulo, Hucitet, 1999.

POSSAS, Mário L. Concorrência schumpeteriana. In: Economia industrial:

Fundamentos teóricos e práticos no Brasil. David Kupfer e Lia Hasenclever (Org.). Rio de Janeiro, Editora Campus, 2002.

RESENDE, Anne Carolina Costa. OLIVEIRA, Ana Maria Hermeto Camilo de. Avaliando Resultados de um Programa de Transferência de Renda: o impacto do Bolsa-Escola sobre os gastos das famílias brasileiras. Revista Estudo Econômico, v. 38, n. 2 2008.

SDPI. Secretaria de Desenvolvimento e Promoção do Investimento do Rio Grande do Sul. Plano de Implantação da Política Industrial: Desenvolvimento Econômico do RS. 2013. Disponível em http://www.sdpi.rs.gov.br/

SCHERER, Frederic; ROSS, Devid. Industrial market structure and economic performance. Boston, Houghton Mifflin, 1990.

SCHIAVO, Camila. Um Estudo sobre o Arranjo Produtivo Audiovisual no Rio Grande do Sul no Contexto da Economia da Cultura. 2015.82 f. Monografia (Bacharelado em Economia) - Faculdade de Ciências Econômicas, Universidade Federal do Rio Grande do Sul, Porto Alegre, 2015. Disponível em:

http://www.lume.ufrgs.br/bitstream/handle/10183/130370/000977059.pdf?sequence $=1$

SUZIGAN, Wilson; FURTADO, João; GARCIA, Renato; SAMPAIO, Sérgio. Clusters ou Sistemas Locais de Produção: mapeamento, tipologia e sugestões de políticas.

Revista de Economia Política, v.24, n.2, outubro/dezembro 2004.

TATSCH, Ana Lúcia; PASSOS, Maria Cristina. Políticas para promoção de arranjos produtivos e inovativos locais no Rio Grande do Sul: os casos dos arranjos de máquinas e implementos agrícolas e de móveis. In: CASSIOLATO, José E.; LASTRES, Helena M. M.; STALLIVIERI, Fabio. (Orgs.). Arranjos produtivos locais: uma alternativa para o desenvolvimento. Experiência de política, volume 2. Rio de Janeiro: E-papers, 2008.

TIROLE, Jean. The theory of industrial organization. Cambridge, 1988.

VIAN, Carlos Eduardo de F.; ANDRADE JÚNIOR, Adílson Martins; BARICELO, Luís Gustavo; SILVA, Rodrigo Peixoto. Origens, evolução e tendências da indústria de máquinas agrícolas. Revista de Economia e Sociologia Rural, Vol.51, № 4, p. 719-744, Out./Dez 2013. 
Suamy Cristina Savaris Linhares. Mestra em Economia Aplicada pela Universidade Federal de Pelotas (UFPEL). Professora Colaboradora da Universidade do Estado de Santa Catarina (UDESC). E-mail para contato: suamy_linhares3@hotmail.com

André Carraro. Doutor em Economia. Professor Associado II (PPGOM/UFPel). Pesquisador do Programa de Pós-Graduação em Organizações e Mercados (PPGOM). Mail: andre.carraro@gmail.com. Endereço: Rua Gomes Carneiro, 1. CEP: 96.010-610; Pelotas-RS. 